\title{
Analyse transversale des projets de recherches participatives réalisées au Consortium régional de recherche en éducation entre 1998 et 2016
}

Par Catherine Dumoulin et Julie Courcy, Université du Québec à Chicoutimi

Résumé

Chaque année, le Consortium régional de recherche en éducation lance un appel de projets invitant les professeurs-chercheurs de I'Université du Québec à Chicoutimi et les acteurs des milieux scolaires du Saguenay-Lac-Saint-Jean, de la Côte-Nord ou de Charlevoix à s'unir afin d'étudier une problématique rencontrée dans un milieu de pratique, dans une perspective de réponse aux besoins des milieux scolaires. Notre article présente les résultats de l'analyse chronologique de 127 projets de recherches participatives soutenues financièrement par le Consortium au cours des 18 dernières années. Nous identifions les problématiques récurrentes, les principales visées des projets, les secteurs de formation les plus impliqués, etc. Cette analyse permet d'une part, de poser un regard éclairé sur l'évolution des pratiques éducatives de collaboration sur le territoire et d'autre part, de fournir des pistes de structuration et de développement pour le Consortium.

Mots-clés: Recherche participative, pratiques éducatives de collaboration, problématiques régionales, pistes de structuration et de développement 


\section{REVUE HYBRIDE DE L'ÉDUCATION}

\section{Le Consortium de recherche en éducation du Saguenay-Lac-Saint- Jean}

Le Consortium régional de recherche en éducation (CRRE) est une initiative des professeurs du département des sciences de l'éducation de I'Université du Québec à Chicoutimi (UQAC). Au cours des 18 dernières années, le Consortium a développé son propre modèle de recherche participative avec l'ensemble des milieux éducatifs du Saguenay-Lac-St-Jean (SLSJ). À ce jour, les dix partenaires ${ }^{1}$ ont investi plus de $500000 \$ 2$ pour la réalisation de plus de 100 projets de recherche participative visant à répondre aux besoins de développement professionnel des praticiens. La valeur ajoutée du CRRE tient plus particulièrement à sa visée d'accompagnement des pratiques professionnelles, grâce à sa capacité éprouvée à travailler en synergie avec les décideurs, les chercheurs et les praticiens, ce qui a contribué à assurer, au fil du temps, la pérennité du lien entre les chercheurs de I'UQAC et les milieux scolaires du SLSJ. Les activités du CRRE s'appuient systématiquement sur les besoins des praticiens ou sur des enjeux prioritaires identifiés au plan régional. En d'autres termes, le CRRE milite depuis 1998 pour que la recherche en région soit moins morcelée, moins isolée, mieux soutenue, mise en valeur, qu'elle ait davantage de retombées sur les pratiques éducatives et que la culture développée permette d'évaluer plus efficacement les pratiques pour les ajuster ou les transformer, tout en développant des modèles qui tiennent compte des changements actuels en matière d'éducation (CRRE, s.d.).

Concrètement, chaque année, le CRRE lance un appel de projets invitant les professeurs-chercheurs de l'UQAC et les acteurs des milieux scolaires du SLSJ, de la Côte-Nord ou de Charlevoix à s'unir afin d'étudier une problématique rencontrée dans un milieu de pratique, dans une perspective de réponse aux besoins. Qu'il s'agisse de l'éducation

\footnotetext{
${ }^{1} 10$ partenaires : UQAC, quatre commissions scolaires et cinq cégeps de la région.

2 Chaque partenaire investit la somme de $1 \$$ par étudiant pour financer la rechercheformation.
} 


\section{REVUE HYBRIDE DE L'ÉDUCATION}

préscolaire, de l'enseignement primaire, secondaire, collégial, universitaire ou professionnel, de l'éducation aux adultes en passant par la formation continue des enseignants en exercice, tous les niveaux d'enseignement sont visés par l'un ou l'autre des axes de subventions proposés par le CRRE.

À notre avis, l'apport du CRRE au développement professionnel des milieux scolaires se traduit par (a) une compréhension plus fine de la situation problématique rencontrée, (b) une adéquation des outils et méthodes pour résoudre la problématique, (c) une amélioration des pratiques professionnelles par une démarche itérative, (d) une implication des praticiens aux changements sociaux par la production de savoirs, d'analyse et de questions de recherche et (e) l'approfondissement chez les praticiens d'une littératie scientifique. Inversement, la collaboration avec les milieux scolaires offrent l'opportunité aux chercheurs du CRRE (a) de travailler à partir des préoccupations des acteurs de leur territoire, (b) de faire connaitre le rôle et le travail du chercheur, (c) de décloisonner le monde des praticiens et des chercheurs, (d) de mettre en place un espace de diffusion des résultats, (e) de développer un réseau de praticiens, (f) de créer des liens actuels entre la théorie et la pratiques dans les activités d'enseignement universitaires et $(\mathrm{g})$ de contribuer au démarrage des activités de recherche des nouveaux professeurs. Au plan régional, la présence d'habitudes de travail entre chercheurs et acteurs du milieu scolaire permet aux décideurs d'avoir une vision d'ensemble des problématiques de leur territoire et d'agir de façon soutenue et directe pour favoriser la réussite éducative.

En 2014, l'idée d'une rétrospective des projets financés par le CRRE au cours des années 1998 à 2016 a émergé. Deux ans plus tard, ce texte propose une rétrospective des projets soumis annuellement au CRRE, incluant le type de demande déposée, la visée des projets, l'emplacement géographique où les projets ont été conduits et le ou les niveau(x) d'enseignement ciblé(s) par les projets. Au final, cette analyse permet d'une part, de poser un regard éclairé sur l'évolution des projets 


\section{REVUE HYBRIDE DE L'ÉDUCATION}

de recherche collaboratifs sur le territoire et d'autre part, de fournir des pistes de structuration et de développement pour le CRRE.

\section{La recherche participative comme fondement théorique}

Ce sont les recherches participatives qui sont au cœur des préoccupations du Consortium. Bien qu'il existe plusieurs formes de recherche participative (recherche-intervention, recherche-formation, recherche-action, recherche impliquée, recherche collaborative), elles présentent toute une similitude, à savoir la prise en compte des savoirs pratiques dans la production de connaissances. Les approches participatives impliquent un processus cyclique comprenant les étapes de collecte des données dans une rétroaction constante, ce qui permet d'accompagner les praticiens, d'évaluer les résultats et de modifier les actions. Anadón (2007) relève certaines caractéristiques communes aux différentes approches participatives: elles s'appliquent à résoudre des problèmes préoccupants des groupes et des personnes intervenant afin de les transformer; elles cherchent à établir un rapport dialectique entre connaissance et action, prenant en compte l'interaction entre la recherche et l'action; elles cherchent la participation active et démocratique des acteurs sociaux et facilitent leur prise de position dans l'espace public; elles rejettent toute forme de rapport hiérarchique entre chercheurs et participants, exigeant une relation d'égalité et d'équité; elles sont considérées comme un outil intellectuel au service de la population; elles constituent une proposition méthodologique utilisant les techniques de collecte et d'analyse des données de la recherche sociale; elles cherchent à briser la dichotomie entre recherche et action, sa méthodologie impliquant la pensée réflexive sur l'action et la tentative d'influer sur l'organisation et la mobilisation des groupes engagés (Anadón, 2007, p. 23-24).

La recherche participative développée au CRRE entraine donc les chercheurs et les acteurs des milieux scolaires dans un processus de co- 


\section{REVUE HYBRIDE DE L'ÉDUCATION}

construction de connaissances valides scientifiquement et utiles pour la communauté professionnelle.

Certaines conditions sont toutefois nécessaires afin de conduire des recherches participatives. Le financement serait la principale barrière à la réalisation de recherches participatives (Savan, Flicker, Kolenda et Mildenberger, 2009) puisqu'il est généralement attribué aux universités, excluant les organisations de la communauté, ce qui peut créer un déséquilibre dans la relation de collaboration. D'ailleurs, peu de bailleurs de fonds sont intéressés ou équipés pour financer à la fois la recherche et l'action (Longtin, 2010). De plus, ce type d'expériences de recherche repose sur le développement de relations de confiance entre les communautés professionnelles et les chercheurs (Jacobson, PruittChapin et Rugeley, 2009) ce qui se construit au cours du travail entre les membres de l'équipe (Klein, 2007). Selon René, Laurin et Dallaire (2009, p.54), « la durée de la démarche facilite la relation de confiance, voire de solidarité, nécessaire au croisement et à la confrontation des savoirs expérientiels individuels afin de former un savoir partagé ».

Le CRRE centre les projets de recherche qu'il soutient autour de quatre axes principaux : la compréhension d'un phénomène ou d'un problème relatif à l'éducation, le développement instrumental d'outils ou de matériel de formation, la résolution de problèmes et la transformation de pratiques. Ainsi, les travaux privilégiés doivent favoriser "l'engagement continu des praticiens et des chercheurs permet[tant] la réalisation conjointe de la recherche, du début à la fin » (CRRE, s.d.). Certes, le projet de recherche présenté peut être initié par un membre de la commission scolaire, un enseignant, un conseiller pédagogique ou un chercheur. Toutefois, le projet doit obligatoirement s'inscrire dans un contexte collaboratif et permettre la participation et la contribution des praticiens de l'éducation et des chercheurs universitaires. La « coconstruction des savoirs avec les acteurs concernés " (Bellot et Rivard, 2013) est donc l'un des principes fondamentaux de la recherche participative soutenus par le CRRE. 


\section{REVUE HYBRIDE DE L'ÉDUCATION}

\section{La méthodologie}

Une méthodologie descriptive a été privilégiée dans cette analyse, ce qui permet notamment de décrire et de présenter concrètement les résultats de cette rétrospective. Afin de dresser un portrait juste et éclairant des projets de recherche proposés au CRRE, nous avons procédé à un inventaire des 127 demandes de projets soumises au CRRE entre les concours de 1997-1998 et de 2015-2016. Chaque demande a été numérisée pour ensuite entamer le processus d'extraction des données brutes.

Plusieurs catégories ont par la suite été créées à partir des sections déjà présentes dans le formulaire de demande de subvention proposé par le Consortium. Les catégories et sous-catégories suivantes ont été retenues : type de demandes (nouvelle ou renouvellement), visée de la demande (développement, compréhension, transformation ou résolution), emplacement géographique (Saguenay, Lac-Saint-Jean, Haute-Côte-Nord, Québec), niveau d'enseignement impliqué dans le projet (préscolaire, primaire, secondaire, collégial, universitaire, formation professionnelle, enseignement aux adultes, formation continue des enseignants). L'ensemble des sous-catégories nous a d'ailleurs permis de trier les données avec plus de justesse. Finalement, c'est à partir de la liste des collaborateurs (et de leurs adresses) ou des écoles participantes fournies comme critères obligatoires dans les formulaires que nous avons été en mesure de préciser les territoires où se sont déroulés les projets de collaboration proposés.

\section{L’analyse des données}

À la lumière des considérations théoriques et méthodologiques, cette section vise à dresser le portrait des projets de recherche soumis au CRRE. Tout d'abord, précisons qu'une fois les projets distribués sur une ligne du temps (Figure 1), il ne semble pas possible de déterminer une tendance du nombre de demandes de projets soumis d'une année à l'autre. En effet, un nombre record maximal de 12 projets ont été soumis 


\section{$\&$}

\section{REVUE HYBRIDE DE L'ÉDUCATION}

en 2004-2005 pour un minimum de trois seulement, en 2013-2014. La Figure 1 démontre aussi la grande variation des projets soumis d'une année à l'autre, et ce, de façon plutôt aléatoire. Par exemple, quatre projets ont été proposés en 2006-2007, puis l'année suivante, 10 projets ont été soumis. En bref, il est possible de dire que le CRRE a pu soutenir en moyenne sept projets annuellement. Nous remarquons toutefois que depuis 2008, le nombre de projets présentés au CRRE pour un soutien financier semble s'être stabilisé autour de la moyenne.

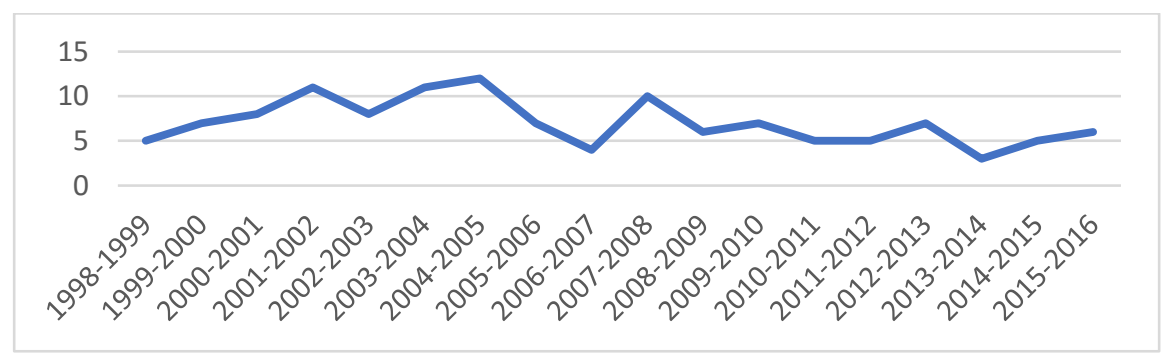

Figure 1. Nombre total de projets soumis au CRRE par année. L'abscisse représente l'année de la demande et l'ordonnée, le nombre de projets présentés au CRRE.

\section{Type de demandes}

Nous avons rapidement constaté que la majorité des projets présentés au CRRE (90/127 ou $71 \%)$ constituait de nouvelles demandes d'appui financier (Tableau 1). En effet, $29 \%$ des projets sont des demandes de renouvellement ayant bénéficié, au cours d'une année antérieure, d'un appui financier du CRRE. Notons qu'un même projet peut en effet être soutenu financièrement par le Consortium durant deux ou trois ans, tout dépendant de l'ampleur de celui-ci. Le ou les responsables de projet doivent soumettre une demande en ce sens, chaque année. Ainsi, depuis 2009, le CRRE a financé presque exclusivement de nouveaux projets : seulement deux demandes de renouvellement ont été déposées, bien que la moyenne annuelle de projet soit demeurée sensiblement la même. 


\section{$\&$}

\section{REVUE HYBRIDE DE L'ÉDUCATION}

Entre 2001-2002 et 2005-2006, il a été possible de constater qu'un nombre plus élevé de demandes de renouvellement ont été déposées au CRRE. En effet, une moyenne de 4,4 demandes de renouvellement a été calculée pour cette période versus une moyenne totale de 1,8. En contrepartie, les nouvelles demandes sont quant à elles demeurées plutôt stables durant cette période, soit une moyenne de 5,2 par rapport à la moyenne totale de 5 . Nous croyons que le nombre élevé de demandes de renouvellement peut expliquer le nombre plus élevé de projets pour cette période, soit entre 8 et 12 pour une moyenne de 9,8 projets par année.

\section{Visées des projets}

Dans le cadre du concours annuel de subvention, les responsables du projet doivent présenter une demande liée à au moins une des quatre visées principales soutenues par le Consortium: le développement instrumental, la compréhension d'un phénomène, la transformation d'une pratique ou la résolution de problème. Nous avons constaté que sur 127 projets, dont 125 où l'information est disponible, 97 projets sont à visée unique. Ainsi, 28 projets s'inscrivent dans plus d'une catégorie, soit deux ou trois d'entre elles. II est possible de constater dans le tableau 2 que les projets visant la transformation des pratiques sont les plus populaires (76/125 ou $60,8 \%$ ), suivis par les projets abordant la compréhension d'un phénomène (45/125 ou $36 \%)$. Moins du quart des projets (27/125 ou $21,6 \%$ ) abordent le développement instrumental et la résolution d'un problème dans la pratique (20/125 ou $16 \%)$. 


\section{REVUE HYBRIDE DE L'ÉDUCATION}

Tableau 1.

Nombre de projets soumis au CRRE par année (Pr/année) selon le type de demande coché sur le formulaire (nouvelle demande (Nv) ou renouvellement $(R)$ )

\begin{tabular}{|c|c|c|c|c|c|c|c|c|c|c|c|c|c|c|c|c|c|c|c|}
\hline Année & 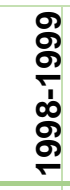 & 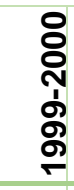 & $\begin{array}{l}\text { ㅇ } \\
\text { ஸे } \\
\text { ঠे } \\
\text { ্ }\end{array}$ & 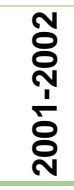 & $\begin{array}{l}\text { ర్ } \\
\text { ণ্} \\
\text { సે } \\
\text { ઠ્స }\end{array}$ & 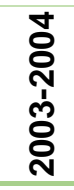 & $\begin{array}{l}\text { 옹 } \\
\text { సे } \\
\text { षे }\end{array}$ & 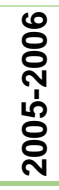 & 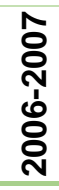 & 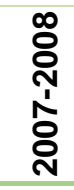 & 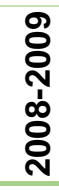 & 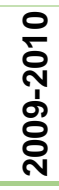 & 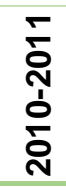 & 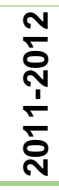 & 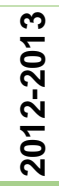 & 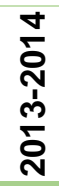 & 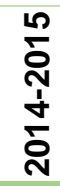 & 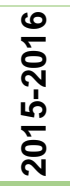 & $\begin{array}{l}\bar{\pi} \\
\text { ㅇ } \\
\end{array}$ \\
\hline Pr/année & 5 & 7 & 8 & 11 & 8 & 11 & 12 & 7 & 4 & 10 & 6 & 7 & 5 & 5 & 7 & 3 & 5 & 6 & 127 \\
\hline Nv & 5 & 4 & 7 & 6 & 3 & 6 & 8 & 3 & 2 & 8 & 4 & 6 & 4 & 5 & 6 & 3 & 4 & 6 & 90 \\
\hline $\mathbf{R}$ & 0 & 2 & 1 & 5 & 5 & 5 & 3 & 4 & 2 & 1 & 2 & 0 & 1 & 0 & 0 & 0 & 1 & 0 & 32 \\
\hline Inconnu & 0 & 1 & 0 & 0 & 0 & 0 & 1 & 0 & 0 & 0 & 0 & 1 & 0 & 0 & 1 & 0 & 0 & 0 & 4 \\
\hline
\end{tabular}




\section{REVUE HYBRIDE DE L'ÉDUCATION}

\section{Tableau 2.}

Nombre de projets pour chaque catégorie de visées

\begin{tabular}{|l|l|}
\hline Catégories de projets subventionnés & Nombre de projets \\
\hline Développement instrumental & 27 \\
\hline Compréhension d'un phénomène & 45 \\
\hline Transformation des pratiques & 76 \\
\hline Résolution de problèmes & 20 \\
\hline Inconnu & 2 \\
\hline
\end{tabular}

\section{Emplacement géographique}

Sur le plan de l'emplacement géographique où se sont déroulés les différents projets annoncés (voir tableau 3), force est de constater qu'une forte majorité (90/125) a été réalisée uniquement sur le territoire du Saguenay. 21 projets sur 125 ont eu lieu au Lac-Saint-Jean seulement, tandis qu'un seul a été conduit sur la Haute-Côte-Nord, plus précisément en collaboration avec le Cégep de Sept-Îles. Précisons que $10,4 \%$ des projets (13/125) ont été conduits sur plus d'un territoire, soit au Saguenay et au Lac-Saint-Jean ou au Saguenay et à Québec. 


\section{REVUE HYBRIDE DE L'ÉDUCATION}

Tableau 3.

Résumé du nombre de projets par territoire géographique

\begin{tabular}{|l|l|}
\hline Territoires & Nb. projets \\
\hline Saguenay & 90 \\
\hline Lac-Saint-Jean & 21 \\
\hline Sept-Îles & 1 \\
\hline Saguenay et Lac-Saint-Jean & 11 \\
\hline Saguenay et Québec & 2 \\
\hline Inconnu & 2 \\
\hline Total & 127 \\
\hline
\end{tabular}

\section{Niveau(x) d'enseignement abordé(s) par les projets de recherche}

Les projets présentés doivent nécessairement être liés à un ou plusieurs niveaux d'enseignement. Les sous-catégories suivantes ont donc été créées: l'éducation préscolaire, l'enseignement primaire, secondaire, professionnel, collégial ou universitaire, l'éducation aux adultes ou la formation continue des enseignants en exercice. Bien que $28,8 \%(36 / 127)$ des projets soumis ne se concentrent qu'à un seul niveau, la majorité des problématiques soutenues par les projets s'intéressent à plus d'un niveau d'enseignement. Par exemple, le projet de Gagnon (2003-2004) portant sur l'Impact de l'éveil aux langues sur la discrimination auditive et sur la motivation à apprendre une langue seconde chez des élèves d'un milieu homogène unilingue s'intéresse à l'expérience des élèves du préscolaire et du primaire. Ou encore, 


\section{REVUE HYBRIDE DE L'ÉDUCATION}

Duquette et Ouellette (2012-2013) qui s'intéressent aux élèves de la fin du secondaire et aux attentes du niveau collégial afin de faciliter la passation d'un ordre d'enseignement à l'autre. C'est ce que démontre le tableau ci-dessous.

Les niveaux scolaires les moins ciblés par les projets de recherche sont l'enseignement aux adultes (5), le secteur de la formation professionnelle (8), l'enseignement universitaire (16) et l'éducation préscolaire (18). Quant à l'enseignement secondaire (31) et collégial (29), ce sont près du quart des projets qui s'intéressent à ces niveaux. Ce sont finalement l'enseignement primaire (62) et la formation continue des enseignants (69) qui ont fait l'objet du plus grand nombre de demandes de financement depuis 1998.

Bien qu'aucune tendance ne puisse être précisée, remarquons qu'en près de 10 ans, soit entre 2005-2006 et 2014-2015, aucun projet n'a ciblé directement l'éducation préscolaire. De même que de 1999-2000 à 2006-2007, aucun projet n'a ciblé l'enseignement aux adultes (EA) et la formation professionnelle (FP). En outre, il est possible d'observer un certain débalancement entre les niveaux abordés par les projets soumis au CRRE. En effet, un peu plus de la moitié des projets soumis (69/127) s'attardent à la formation continue des enseignants en cours de pratique ou à l'enseignement primaire (62/127). En contrepartie, 47 projets sur 127 abordent soit l'enseignement aux adultes, la formation professionnelle, l'enseignement universitaire ou l'éducation préscolaire. Ainsi, presque la

moitié des projets soutenus par le Consortium s'effectuent en collaboration avec l'enseignement primaire, spécifiquement ou non. 


\section{REVUE HYBRIDE DE L'ÉDUCATION}

Tableau 4.

Nombre de projets soumis par année pour chaque niveau d'enseignement (préscolaire $(P r)$, primaire $(P)$, secondaire $(S)$, collégial (C), universitaire (Uni), enseignements aux adultes (EA), formation professionnelle (FP), formation continue aux enseignants (FC))

\begin{tabular}{|c|c|c|c|c|c|c|c|c|}
\hline Année & $\operatorname{Pr}$ & $\mathbf{P}$ & $S$ & C & Uni & EA & FP & FC \\
\hline 1998-1999 & 1 & 3 & 1 & 1 & 1 & 1 & 0 & 4 \\
\hline 1999-2000 & 1 & 5 & 4 & 1 & 2 & 0 & 0 & 6 \\
\hline $2000-2001$ & 2 & 3 & 2 & 3 & 1 & 0 & 0 & 4 \\
\hline 2001-2002 & 2 & 6 & 0 & 4 & 0 & 0 & 0 & 6 \\
\hline 2002-2003 & 3 & 4 & 0 & 2 & 0 & 0 & 0 & 6 \\
\hline 2003-2004 & 6 & 6 & 2 & 0 & 0 & 0 & 0 & 8 \\
\hline 2004-2005 & 2 & 5 & 5 & 3 & 2 & 0 & 0 & 5 \\
\hline 2005-2006 & 0 & 5 & 0 & 2 & 2 & 0 & 0 & 4 \\
\hline 2006-2007 & 0 & 2 & 0 & 2 & 0 & 0 & 0 & 1 \\
\hline $2007-2008$ & 0 & 4 & 4 & 1 & 2 & 0 & 2 & 6 \\
\hline 2008-2009 & 0 & 4 & 2 & 1 & 2 & 0 & 0 & 3 \\
\hline 2009-2010 & 0 & 2 & 0 & 2 & 1 & 2 & 1 & 3 \\
\hline $2010-2011$ & 0 & 2 & 1 & 2 & 0 & 1 & 1 & 3 \\
\hline 2011-2012 & 0 & 2 & 2 & 1 & 1 & 0 & 0 & 2 \\
\hline 2012-2013 & 0 & 4 & 4 & 1 & 0 & 1 & 1 & 4 \\
\hline 2013-2014 & 0 & 0 & 1 & 0 & 0 & 0 & 2 & 2 \\
\hline 2014-2015 & 0 & 4 & 2 & 0 & 2 & 0 & 0 & 1 \\
\hline $2015-2016$ & 1 & 1 & 1 & 3 & 0 & 0 & 1 & 1 \\
\hline Total & 18 & 62 & 31 & 29 & 16 & 5 & 8 & 69 \\
\hline
\end{tabular}




\section{REVUE HYBRIDE DE L'ÉDUCATION}

\section{Conclusion}

L'analyse transversale des projets de recherche participative réalisés au CRRE entre 1998 et 2016 avait pour objectifs d'identifier les problématiques récurrentes, les principales visées des projets, les secteurs de formation les plus impliqués, etc. C'est donc en cohérence avec nos objectifs de départ que cet article a pu présenter une description des projets de recherche soutenus par le CRRE. II a été possible de constater qu'en moyenne, sept projets sont proposés annuellement au Consortium et que la majorité de ces demandes de soutien financier sont de nouveaux projets. Les projets visant la transformation des pratiques semblent les plus populaires. Nous croyons que ce type de projet rejoint davantage les praticiens sur le terrain puisqu'il suppose un résultat concret, un changement, voire l'amélioration de leur pratique. Sur le plan géographique, l'emplacement de I'UQAC a certainement un effet sur la conduite des projets en sol majoritairement saguenéen, et ce, malgré l'ouverture vers le Lac-Saint-Jean.

II est encourageant pour la recherche participative régionale de constater que la majorité des projets intègrent divers niveaux d'enseignement et différents milieux de pratique. Les savoirs se construisent à partir de l'expertise des acteurs de terrains variés et c'est précisément ce que le Consortium souhaite. Les chercheurs et les praticiens travaillent en collégialité, vers des buts déterminés en équipe, visant d'un côté la production de connaissances et de l'autre, le développement ou la transformation de ses pratiques (CRRE, s.d.). Ce constat est un bon indicateur que la collaboration, l'échange et le partage entre milieux de pratique et de recherche permettent d'enrichir et d'accroitre les connaissances en plus de favoriser la mise en place de nouvelles pratiques éducatives. En outre, il s'avère intéressant de se questionner sur ce que le Consortium peut faire pour encourager les chercheurs et les praticiens des milieux moins sollicités par la recherche participative à présenter des demandes de projets. Est-ce que les axes proposés rejoignent leur réalité? Est-ce que les fonds sont suffisants pour 


\section{REVUE HYBRIDE DE L'ÉDUCATION}

permettre aux équipes de réaliser des recherches sur un territoire éloigné?

Tel que nous l'avons vu, certaines années se sont démarquées par le nombre important de demandes de renouvellement, ce qui a eu un effet sur le nombre total annuel de projets soumis. Ainsi, constatant que peu de demandes de renouvellement ont été proposées au CRRE depuis 2009, il est possible de s'interroger sur la longévité des projets ou sur la perspective à long terme envisagée par les responsables de projet. Dans un contexte économique instable, tant sur le plan du financement des universités que socialement, est-ce que le Consortium pourrait trouver une stratégie pour encourager l'initiation de projets de recherche axés sur la collaboration, à plus long terme? Cette baisse des projets soumis à des fins de renouvellement pourrait-elle s'expliquer par le fait que le CRRE offre un tremplin important aux nouveaux projets pour des demandes de financement plus substantiels sur la scène provinciale et nationale? Nous pourrions peut-être aussi risquer un lien entre le nombre de demandes de renouvellement et les attentes des membres du conseil d'administration. De fait, il y a un roulement des membres siégeant au conseil d'administration, ce qui peut influencer leur choix de valoriser ou non les demandes de renouvellement. En terminant, nous croyons fortement que même après une année de soutien financier, la recherche participative encouragée par le CRRE peut avoir eu les résultats escomptés pour le chercheur et pour le praticien et ainsi, ouvrir la porte à de nouvelles demandes de projet. 


\section{REVUE HYBRIDE DE L'ÉDUCATION}

\section{Références}

Anadón, M. (dir.). (2007). La Recherche participative : multiples regards. Québec, QC : Presses universitaires du Québec.

Bellot, C. et Rivard, J. (2013). La reconnaissance : Un enjeu au cœur de la recherche participative. Nouvelles pratiques sociales, 25(2), 105124.

Consortium régional de recherche en éducation (CRRE). (2016). À propos. Repéré à http://etunix.uqac.ca/crre/?page id=2

Jacobson, M., Pruitt-Chapin, K. et Rugeley, C. (2009). Toward Reconstructing Poverty Knowledge: Addressing Food Insecurity through Grassroots Research Design and Implementation. Journal of Poverty, 13, 1-19.

Klein, J.-L. (2007). La recherche- action en développement local : possibilités et contraintes. Dans M. Anadón (dir.), La recherche participative: multiples regards (p.31-45). Québec, QC : Presses de l'Université du Québec.

Longtin, D. (2010). Revue de la littérature: la recherche-action participative, le croisement des savoirs et des pratiques et les incubateurs technologiques de coopératives populaires (Rapport no ET1102). Montréal, QC : Centre de recherche sur les innovations sociales.

René, J.-F., Laurin I. et Dallaire, N. (2009). Faire émerger le savoir d'expérience de parents pauvres : forces et limites d'une recherche participative. Recherches qualitatives, 28(3), 40-63.

Savan, B., Flicker, S., Kolenda, B. et Mildenberger, M. (2009). How to facilitate (or discourage) community - based research: recommendations based on a Canadian survey. Local Environment, 14(8), Septembre 2009, 783-796. 